\title{
Optimizing the Jiles-Atherton Model of Hysteresis by a Genetic Algorithm
}

\author{
Peter R. Wilson, Member, IEEE, J. Neil Ross, and Andrew D. Brown, Senior Member, IEEE
}

\begin{abstract}
Modeling magnetic components for simulation in electric circuits requires an accurate model of the hysteresis loop of the core material used. It is important that the parameters extracted for the hysteresis model be optimized across the range of operating conditions that may occur in circuit simulation. This paper shows how to extract optimal parameters for the Jiles-Atherton model of hysteresis by the genetic algorithm approach. It compares performance with the well-known simulated annealing method and demonstrates that improved results may be obtained with the genetic algorithm. It also shows that a combination of the genetic algorithm and the simulated annealing method can provide an even more accurate solution than either method on its own. A statistical analysis shows that the optimization obtained by the genetic algorithm is better on average, not just on a one-off test basis. The paper introduces and applies the concept of simultaneous optimization for major and minor hysteresis loops to ensure accurate model optimization over a wide variety of operating conditions. It proposes a modification to the Jiles-Atherton model to allow improved accuracy in the modeling of the major loop.
\end{abstract}

Index Terms-Circuit simulation, genetic algorithm, hysteresis, Jiles-Atherton, magnetic component modeling, optimization.

\section{INTRODUCTION}

$\mathbf{T}$ RANSFORMERS and inductors are essential components in a wide variety of power and communications applications, and the accurate modeling of these devices for use in circuit simulation is essential to predict design performance. It is required to accurately represent the hysteresis behavior of the magnetic core material used in these components in the simulation model. One model that has been used quite widely is that of Jiles and Atherton [1], [2]. Jiles et al. [7] show how the parameters for the model may be extracted from a set of measured data for a major hysteresis loop but do not consider arbitrary loop sizes, and Prozygy [8] has established the effects of parameter variations on the major loop. Optimization methods applied to fit the Jiles-Atherton hysteresis loops to measured data have been investigated by Schmidt and Guldner [9], and Lederer et al. [10] using the well-known simulated annealing approach. Genetic algorithms provide an alternative approach to optimization which may have some advantages, especially when considering the more complex problem of fitting several loops simultaneously.

Manuscript received June 16, 2000. This work was supported in part by the Engineering and Physical Sciences Research Council and Advanced Power Components Ltd., both in the U.K.

The authors are with the Department of Electronics and Computer Science, University of Southampton, Highfield, Southampton, U.K. (e-mail prw99r@ecs.soton.ac.uk; jnr@ecs.soton.ac.uk; adb@ecs.soton.ac.uk).

Publisher Item Identifier S 0018-9464(01)01822-2.
The use of genetic, or evolutionary, algorithms to solve difficult engineering problems is a relatively recent innovation. Holland [3] and Goldberg [4] are two of the pioneers of this technique, and the last $10 \mathrm{yr}$ have seen a plethora of applications for genetic algorithms from systems design to topology analysis [5]. The fundamental difference between genetic algorithms and conventional optimization techniques, such as simulated annealing [6], is that in certain problems, the computational effort involved in a standard exhaustive search method would be prohibitive. The random nature of genetic algorithms may not find the absolute best solution, but it has a greater chance of finding a good solution, quickly, for difficult problems. This randomness also works well for problems with chaotic or ill-defined behavior difficult to classify, and those problems with local maxima or minima that would perhaps trap a conventional search algorithm.

The optimization of magnetic materials is a problem which has aspects suited to the application of genetic algorithms. Even though the Jiles-Atherton [1] model of hysteresis is well understood mathematically, the parameters are interlinked in such a way that the set of possible combinations of parameters may be large. Significant changes in the shape of the hysteresis loop may result from small parameter variations. These two aspects give a relatively high risk of local maxima or minima being found or instabilities in a conventional algorithm. Other approaches for modeling magnetic materials such as the Preisach [11] model have no direct link between behavior and material physical properties so are natural potential targets for the genetic algorithm approach.

\section{OutLine OF ThE GENETIC AlgORIthm Method}

\section{A. Overview}

The flowchart of a generic genetic algorithm is shown in Fig. 1. The algorithm is based on the concept of natural selection. The first step is to define an initial population of individuals, or set of magnetic model parameters, which is the first generation for the algorithm. In this application, the initial population is formed by taking an initial set of parameters which are then subjected to random variations.

\section{B. Choosing Parents}

For each generation, there will be a number of children created by combining the characteristics of two parent individuals. The choice of parents is determined by random and selective methods. First, a set of possible parents is randomly chosen from the current generation. The prospective parents are then ranked 


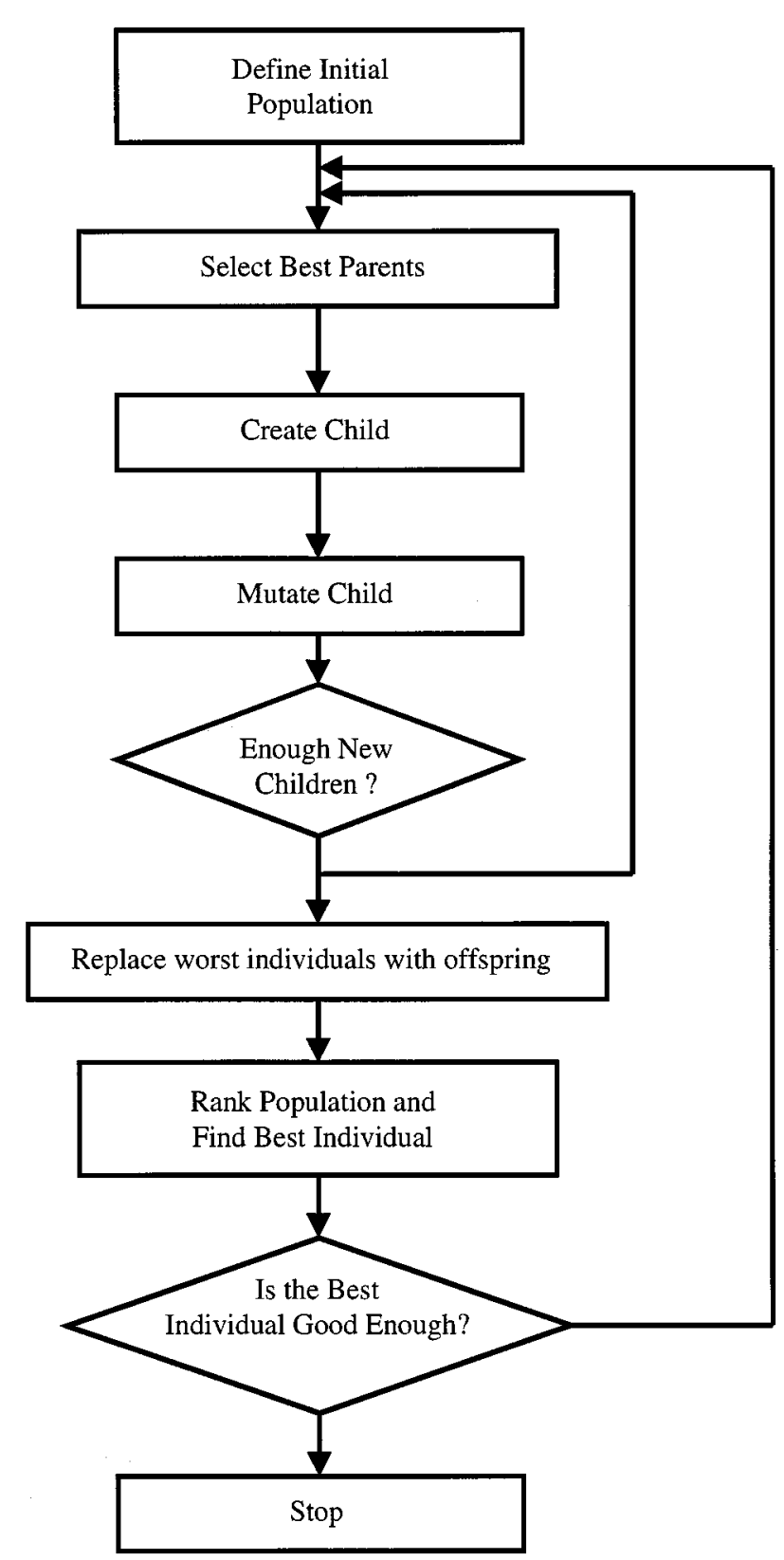

Fig. 1. Generic genetic algorithm method.

in terms of a fitness function to find the best individual, which is then chosen as the first parent. In this case, the fitness function is a measure of how closely the $\mathrm{BH}$ (flux density-B versus magnetic field strength- $\mathrm{H}$ ) curve matches the target. This process is repeated with a new set of possible parents to find the second parent. Once the two parents have been selected, then their characteristics can be combined to create a new individual (child).

\section{Creating New Children}

A new child is created by the random combination of the characteristics of the parents. The overall set of parameters is defined as a string (chromosome) made up of individual parameters (genes). Each parameter is represented by a floating-point binary number. The child's chromosome is then constructed by

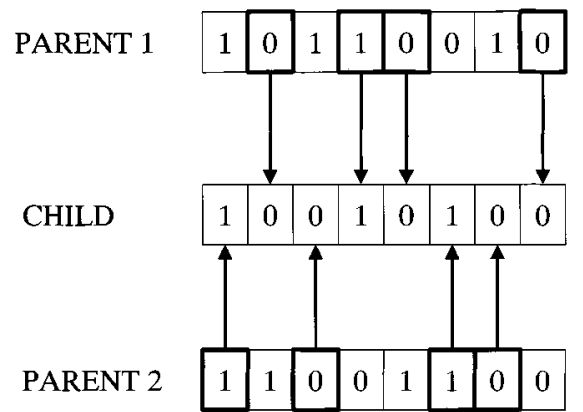

Fig. 2. Genetic algorithm crossover.

combining the genes of the parents using a crossover method, illustrated in Fig. 2. The selection of parents and creation of new individuals (children) is then repeated until the specified number of children is achieved.

As in natural reproduction, there is a risk of mutation during the crossover process. This is implemented by adding random changes to a proportion of the children created.

\section{Renewing the Population}

Once the required number of children has been created, the population as a whole is adjusted, in this case keeping the size of the population constant. To do this, the parent's generation is tested for fitness, ranked, and the worst individuals replaced by the children.

The new generation is again ranked using the fitness function, and the best individual is evaluated to see if it meets the requirements of the goal function, and if so, the algorithm stops; otherwise, the algorithm can continue.

\section{FitNESS OR GOAL FUNCTIONS}

The fitness, or goal, function that defines the performance of the model is based on a simple least squares error approach comparing the curve(s) with the target on a point-by-point basis. An alternative goal function described by Wilson and Ross [12] has been implemented based on performance metrics such as initial permeability, saturation flux, and energy loss. By using weighting of metrics, the goal function is made appropriate for the ultimate application.

\section{OPtIMIZING THE JiLES-ATHERTON MODEL OF HySTERESIS}

\section{A. Jiles-Atherton Model}

The Jiles and Atherton [1], [2] model of hysteresis is a physically based approach for modeling magnetic hysteresis. The parameters of the model are related to physical features in the model such as the saturation magnetization, summarized as follows:

$k \quad$ irreversible loss;

a anhysteretic behavior;

c reversible/irreversible proportions;

$\alpha \quad$ effective field;

$m s \quad$ saturation magnetization. 
Unfortunately, these parameters will only provide an accurate fit for specific sigmoid forms of the hysteresis loop but is inadequate for accurate modeling of early closure of the hysteresis loop or square loops.

\section{B. Improvement of the Jiles-Atherton Model}

There is no fundamental reason why the parameters should be fixed, and they can therefore be made functions of the applied field or the flux density to obtain a better fit with measured data. Jiles and Atherton [2] explain that the parameter which defines the loss $k$ can be made a function of the applied field or the flux density and propose a linear function. It was found, however, that a Gaussian function of the form shown in (1) gave excellent results, and this was therefore used in this paper. This function has the added advantage of no discontinuity around zero when the applied field changes polarity which improves simulation convergence.

$$
k=k_{0} * e^{-\left(H^{2} / 2 \sigma^{2}\right)}
$$

where

$k_{0} \quad$ default value of the $k$ parameter;

$H$ applied field;

$\sigma \quad$ standard deviation of the Gaussian function.

Tests with materials such as 3F3 and N30 have demonstrated a significant improvement in the accuracy of the modeled $\mathrm{BH}$ curves.

\section{Applying the Genetic Algorithm Approach}

A toroid made of the Siemens N30 material was tested, and the resulting $\mathrm{BH}$ loop used for optimization. The Jiles-Atherton model was optimized using the classical model and also the improved model utilizing the variable $k$ parameter. The optimization was carried out using the well-understood simulated annealing method as a control and also with a genetic algorithm approach. The simulated annealing approach was carried out with a variation of $10 \%$, a control factor of 0.001 , and 2500 iterations used. The genetic algorithm used 50 generations of a population including 50 individuals ( 50 times 50 giving a rough equivalent of 2500 iterations). Each generation produced 40 children of whom 20 were mutated. A variation of $10 \%$ was introduced in the mutation process. In each case, the fitness function used the least squares error approach.

The resulting mean errors between the simulated and measured results are summarized in Table I and Fig. 3. Table I shows the error is significantly reduced for the genetic algorithm and that the variable $k$ parameter makes a significant difference for both optimization methods. Fig. 3 shows the error versus the number of iterations and again clearly, the genetic algorithm with the Gaussian modification of $k$ is the best result.

Interestingly, when a combination of simulated annealing and the genetic algorithm was applied, an even better result was achieved. This can be explained with the fact that the two methods have different strengths. The genetic algorithm is very good at finding the correct area of the solution, tolerant of local maxima and minima, and the simulated annealing method is excellent at refining a solution systematically to the nearest maximum or minimum.
TABLE I

JiLES-ATHERTON MOdel PARAMETERS AND ERROR FOR SIMULATED ANNEALING AND GENETIC ALGORITHM OPTIMIZATION

\begin{tabular}{ccccc}
\hline \hline Parameter & $\begin{array}{c}\text { Genetic } \\
\text { Algorithm }\end{array}$ & $\begin{array}{c}\text { Genetic } \\
\text { Algorithm } \\
\text { Gaussian k }\end{array}$ & $\begin{array}{c}\text { Simulated } \\
\text { Annealing }\end{array}$ & $\begin{array}{c}\text { Simulated } \\
\text { Annealing } \\
\text { Gaussian k }\end{array}$ \\
\hline $\mathrm{a}$ & 13.8 & 9.29 & 7.26 & 6.96 \\
$\mathrm{c}$ & 0.799 & 0.497 & 0.150 & 0.308 \\
$\mathrm{k}$ & 11.01 & 12.67 & 7.19 & 11.176 \\
$\alpha$ & $6.78 \mathrm{e}-6$ & $29.1 \mathrm{e}-6$ & $4.29 \mathrm{e}-6$ & $4.54 \mathrm{e}-6$ \\
$\mathrm{M}_{\mathrm{S}}$ & 293318 & 268562 & 264500 & 257479 \\
$\sigma$ & - & 16.55 & - & 15.54 \\
Error & 0.1416 & 0.019 & 0.188 & 0.035 \\
\hline \hline
\end{tabular}

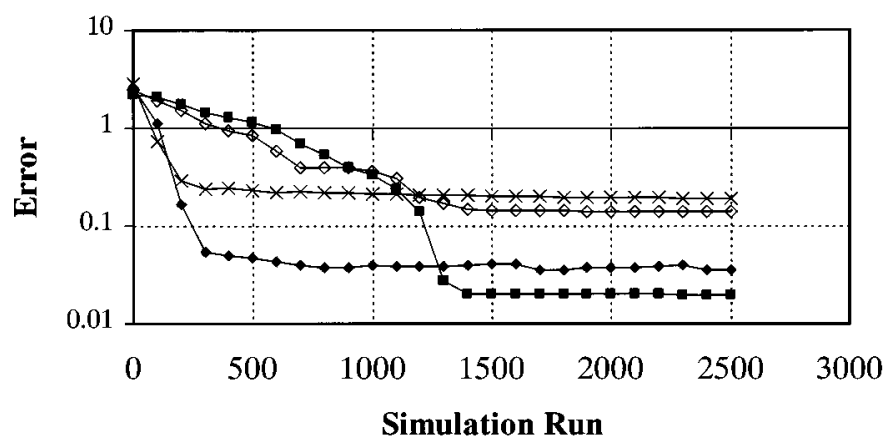

* Simulated Annealing (Original JA Model)

$\multimap$ Genetic Algorithm (Original JA Model)

.- Simulated Annealing (Modified JA Model)

$\rightarrow$ Genetic Algorithm (Modified JA Model)

Fig. 3. Comparison of simulated annealing and genetic algorithm error functions.

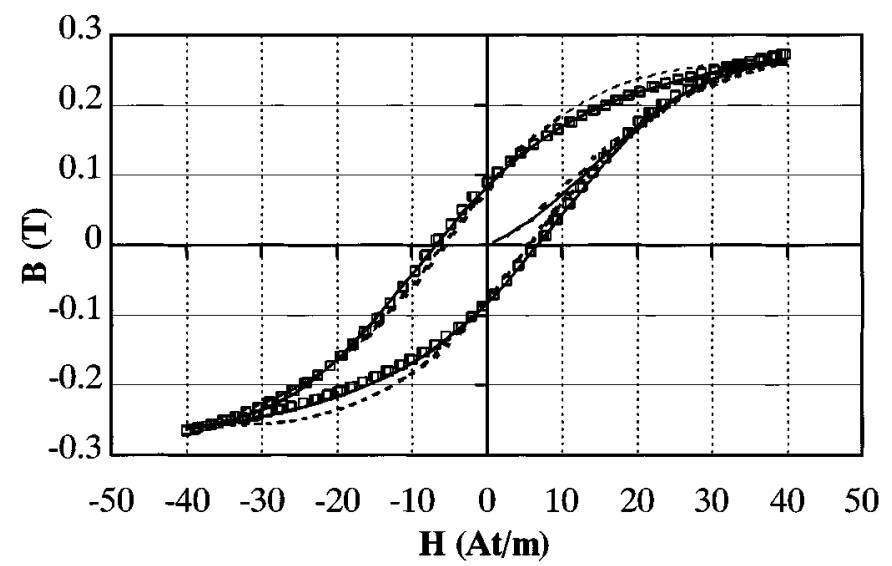

- Measured ..... Original Jiles-Atherton — Modified Jiles-Atherton

Fig. 4. Comparison of measured, simulated annealing and genetic algorithm + simulated annealing $B H$ curves.

The results of the optimization are perhaps best visualized by observing the resulting $B H$ loops. Fig. 4 shows the optimized $B H$ loop using the original Jiles-Atherton model with no modification and also the optimized curve with a Gaussian variation of $k$ using a combination of genetic algorithm and simulated annealing optimization. 


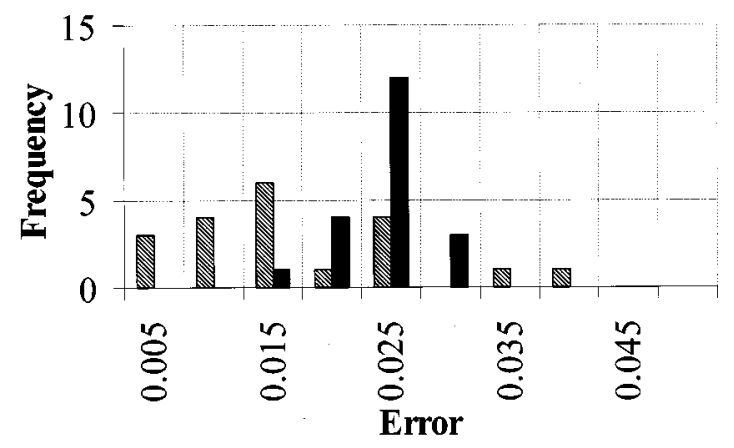

$\mathbb{Q}$ Genetic Algorithm $\square$ Simulated Annealing

Fig. 5. Statistical comparison of the performance of the simulated annealing and genetic algorithm approaches for optimization of the Jiles-Atherton magnetic material model.

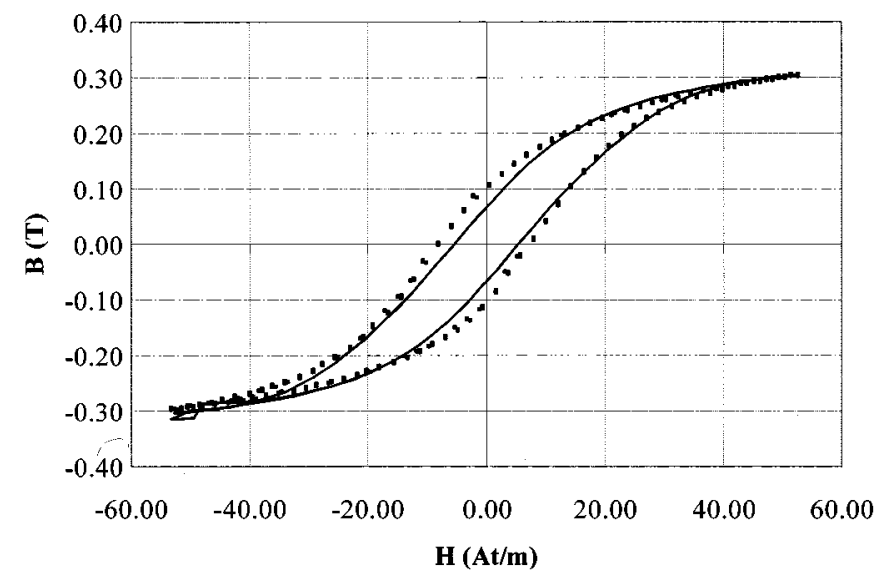

- Measured - Simulated

Fig. 6. Multiple loop optimization results for Siemens N30 (major loop).

\section{STATISTICAL ANALYSIS OF OptiMizaTiOn RESUlTS}

Although the individual optimization results previously shown are encouraging, due to the random nature of the optimization process in both simulated annealing and genetic algorithm approaches, it is appropriate to investigate the performance of the respective methods statistically. The optimizations were therefore repeated over a number of runs (20) and the resulting errors compared. The simulated annealing approach was carried out with a variation of $10 \%$, a control factor of 0.001 , and 2000 iterations used. The genetic algorithm used 40 generations of a population including 50 individuals (40 times 50 giving a rough equivalent of 2000 iterations). Each generation produced 40 children of whom 20 were mutated. A variation of $20 \%$ was introduced in the mutation process. The simulated annealing approach gave a mean value for the error of 0.0219 , with a standard deviation of 0.003 , while the genetic algorithm gave a mean value for the error of 0.0148 , with a standard deviation of 0.009 . Fig. 5 shows the histogram of the respective errors for the two methods.

\section{Multiple LoOP OPTIMIZATION}

In practice, for circuit simulation, the resulting optimized model for a magnetic material must be accurate over a wide

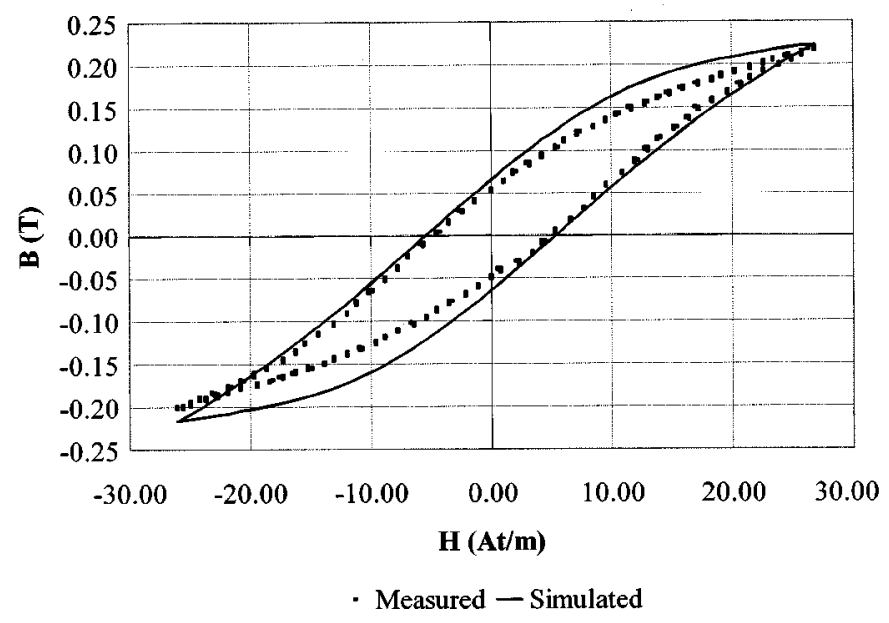

Fig. 7. Multiple loop optimization results for Siemens N30 (medium loop).

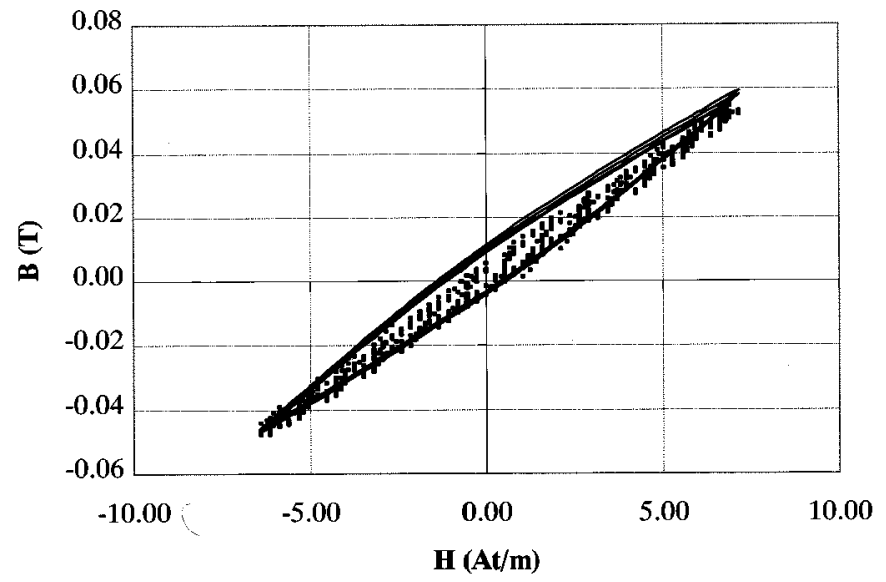

. Measured — Simulated

Fig. 8. Multiple loop optimization results for Siemens N30 (minor loop).

variety of operating conditions. To ensure this is the case, the optimization goal function was extended to allow the optimization of a set of $B H$ loops rather than a single major loop. Each loop in the set has its own weighting, so if it is essential that the minor loop has a high level of accuracy, but the major loop is not significant, then the weighting can be increased for the minor loop accordingly. An example of this is shown in Figs. 6-8, where the minor loop weighting was set to 5 to improve the relative optimization for the smaller loops. The resulting family of curves show a good match for the minor loop, a reasonable match for the major loop, but a poor match for the medium-sized loops.

\section{CONCLUSION}

This paper has demonstrated that it is possible to apply the genetic algorithm technique to the optimization of parameters for the Jiles-Atherton model. It is shown that a small modification to the Jiles-Atherton model gives an improved matching of the major $B H$ loop.

Statistical analysis shows that the genetic algorithm approach provides an optimized model, which is more accurate than using the simulated annealing approach. 
The method of multiple loop optimization is introduced, which shows how models can be optimized for a range of $B H$ loops such as minor, medium, and major loops. This method is appropriate for the optimization of model parameters to be used in circuit simulation where a wide variety of operating conditions occur

\section{REFERENCES}

[1] D. C. Jiles and D. L. Atherton, "Theory of ferromagnetic hysteresis," $J$. Magn. Magn. Mater., vol. 61, pp. 48-60, 1986.

[2] —_, "Theory of ferromagnetic hysteresis," J. Appl. Phys., vol. 55, no. 6, pp. 2115-2120, Mar. 1984

[3] J. H. Holland, Adaption in Natural and Artificial Systems. Ann Arbor, MI: Univ. Michigan Press, 1975.

[4] D. E. Goldberg, Genetic Algorithms in Search, Optimization and Machine Learning. Reading, MA: Addison-Wesley, 1986.

[5] Genetic Algorithms in Engineering Systems: Innovations and Applications. London, U.K.: IEE, Sept. 1997.

[6] P. J. M. Laarhoven and E. H. L. Aarts, Simulated Annealing: Theory and Applications. Boston, MA: Kluwer, 1989.

[7] D. C. Jiles, J. B. Thoelke, and M. K. Devine, "Numerical determination of hysteresis parameters for the modeling of magnetic properties using the theory of ferromagnetic hysteresis," IEEE Trans. Magn., vol. 28, pp. 27-35, Jan. 1992.

[8] S. Prigozy, "PSPICE computer modeling of hysteresis effects," IEEE Trans. Educat., vol. 36, pp. 2-5, Feb. 1993.

[9] N. Schmidt and H. Güldner, "Simple method to determine dynamic hysteresis loops of soft magnetic materials," IEEE Trans. Magn., vol. 32, pp. 489-496, Mar. 1996.

[10] D. Lederer, H. Igarashi, A. Kost, and T. Honma, "On the parameter identification and application of the Jiles-Atherton hysteresis model for numerical modeling of measured characteristics," IEEE Trans. Magn., vol. 35, pp. 1211-1214, May 1999.

[11] F. Preisach, "Über die magnetische nachwirkung," Zeitschrift Fur Physik, pp. 277-302, 1935.

[12] P. R. Wilson and J. N. Ross, "Definition and application of magnetic material metrics in modeling and optimization," IEEE Trans. Magn., submitted for publication.
Peter R. Wilson (M'98) received the B.Eng. degree in electrical and electronic engineering and the Postgraduate Diploma in digital systems engineering from Heriot-Watt University, Edinburgh, U.K., in 1988 and 1992, respectively. He also received the M.B.A. degree from Heriot-Watt University in 1999. Since 1999, he has been pursuing the Ph.D. degree at the University of Southampton, Southampton, U.K

He worked in the Navigation Systems Division of Ferranti plc., Edinburgh, from 1988 to 1990 on fire control computer systems, before moving in 1990 to the Radar Systems Division of GEC-Marconi Avionics, Edinburgh. From1990 to 1994 , he worked on modeling and simulation of power supplies, signal processing systems, and servo and mixed technology systems. From 1994 to 1999 , he worked as European Product Specialist with Analogy Inc., Swindon, U.K During this time, he developed a number of models, libraries, and modeling tools for the Saber simulator, especially in the areas of power systems, magnetic components, and telecommunications. His current research interests include modeling of magnetic components in electric circuits, VHDL-AMS modeling and simulation, and the development of electronic design tools.

Mr. Wilson is a Member of the IEE and a Chartered Engineer.

J. Neil Ross received the B.Sc. degree in physics from the University of St. Andrews, St. Andrews, U.K., in 1970 and the Ph.D. degree in 1974 from the same University for his work on the physics of ion laser discharges.

For 12 years, he worked at the Central Electricity Research Laboratories of the CEGB, undertaking research on the physics of high-voltage breakdown and optical fiber sensors for use in a high-voltage environment. He joined the University of Southampton, Southampton, U.K. in 1987 and has undertaken research in a variety of fields associated with instrumentation and measurement. His current research interests include the modeling of magnetic components for communications, instrumentation, and power applications.

Andrew D. Brown (M'90-SM'96) was born in the United Kingdom in 1955 He received the B.Sc. degree (with honors) in physical electronics and the Ph.D. degree in microelectronics from Southampton University, Southampton, U.K., in 1976 and 1981, respectively.

He was appointed lecturer in electronics at Southampton in 1981, Senior Lecturer in 1989, Reader in 1992, and was appointed to an established chair in 1998 He was a Visiting Scientist at IBM, Hursley Park, U.K., in 1983 and a Visiting Professor at Siemens NeuPerlach, Munich, Germany, in 1989. He is currently Head of the Electronic System Design Group, Electronics Department, Southampton University. The group has interests in all aspects of simulation, modeling, synthesis, and testing.

Dr. Brown is a Fellow of the IEE, a Chartered Engineer, and a European Engineer. 